\title{
THE GROUP OF CAUSAL AUTOMORPHISMS
}

\author{
DO-HYUNG KIM
}

AbStract. The group of causal automorphisms on Minkowski spacetime is given and its structure is analyzed.

\section{INTRODUCTION}

In 1964, Zeeman has shown that any causal automorphism on Minkowski space-time $\mathbb{R}_{1}^{n}$ can be represented by a composite of orthochronous transformation, traslation and homothety when $n \geq 3$.(See Ref. 1]). Therefore, if we let $G$ be the group of all causal automorphisms on $\mathbb{R}_{1}^{n}$ with $n \geq 3$, then $G$ is isomorphic to the semi-direct product of $\mathbb{R}^{+} \times \mathcal{O}$ and $\mathbb{R}_{1}^{n}$ where $\mathbb{R}^{+}$ is the group of positive real numbers and $\mathcal{O}$ is the group of orthochronous transformations on $\mathbb{R}_{1}^{n}$. However, as he remarked, Zeeman's theorem does not hold when $n=2$.

In connection with this, recently, the standard form of causal automorphism on $\mathbb{R}_{1}^{2}$ is given in Ref. 22. In this paper, we improve the result in Ref. 2] and by use of this, the structure of the causal automorphism group is analyzed when $n=2$.

\section{Causal automorphisms on $\mathbb{R}_{1}^{2}$}

The following is a known result.

Theorem 2.1. Let $F: \mathbb{R}_{1}^{2} \rightarrow \mathbb{R}_{1}^{2}$ be a causal automorphism. Then, there exist a continuous functions $g: \mathbb{R} \rightarrow \mathbb{R}$ and a homeomorphism $f: \mathbb{R} \rightarrow \mathbb{R}$ which satisfy $\sup (g \pm f)=\infty, \inf (g \pm f)=-\infty$ and $\left|\frac{g(t+\delta t)-g(t)}{f(t+\delta t)-f(t)}\right|<1$ for all $t$ and $\delta t$, such that if $f$ is increasing, then $F$ is given by $F(x, y)=\left(\frac{f(x-y)+f(x+y)}{2}+\frac{g(x+y)-g(x-y)}{2}, \frac{f(x+y)-f(x-y)}{2}+\frac{g(x-y)+g(x+y)}{2}\right)$ and if $f$ is decreasing, then we have

$F(x, y)=\left(\frac{f(x+y)+f(x-y)}{2}+\frac{g(x-y)-g(x+y)}{2}, \frac{f(x-y)-f(x+y)}{2}+\frac{g(x+y)+g(x-y)}{2}\right)$. Conversely, for any functions $f$ and $g$ which satisfy the above conditions, the function $F: \mathbb{R}_{1}^{2} \rightarrow \mathbb{R}_{1}^{2}$ defined as above, is a causal automorphism.

Proof. See Theorem 4.4 in Ref. [2].

Key words and phrases. Lorentzian geometry, general relativity, causality, Cauchy surface, space-time, global hyperbolicity, causal automorphism, Minkowski space-time. 
We remark that in the above theorem, for given causal automorphism $F(x, y)=\left(F_{1}(x, y), F_{2}(x, y)\right)$, the homeomorphism $f$ and the continuous function $g$ are uniquely determined by $f(t)=F_{1}(t, 0)$ and $g(t)=F_{2}(t, 0)$. By use of this, we improve the above theorem.

Theorem 2.2. Let $F: \mathbb{R}_{1}^{2} \rightarrow \mathbb{R}_{1}^{2}$ be a causal automorphism on $\mathbb{R}_{1}^{2}$. Then, there exist unique homeomorphisms $\varphi$ and $\psi$ of $\mathbb{R}$, which are either both increasing or both decreasing, such that if $\varphi$ and $\psi$ are increasing, then we have $F(x, y)=\frac{1}{2}(\varphi(x+y)+\psi(x-y), \varphi(x+y)-\psi(x-y))$, or if $\varphi$ and $\psi$ are decreasing, then we have $F(x, y)=\frac{1}{2}(\varphi(x-y)+\psi(x+y), \varphi(x-y)-\psi(x+y))$.

Conversely, for any given homeomorphisms $\varphi$ and $\psi$ of $\mathbb{R}$, which are either both increasing or both decreasing, the function $F$ defined as above is a causal automorphism of $\mathbb{R}_{1}^{2}$.

Proof. For any given causal automorphism $F: \mathbb{R}_{1}^{2} \rightarrow \mathbb{R}_{1}^{2}$, by the previous theorem, we can get unique homeomorphism $f$ and unique continuous function $g$. Let $\varphi=f+g$ and $\psi=f-g$. Then, clearly, $\varphi$ and $\psi$ are continuous.

The conditions $\sup (g \pm f)=\infty, \inf (g \pm f)=-\infty$ imply that $\varphi$ and $\psi$ are surjective. If $\varphi(t)=\varphi\left(t^{\prime}\right)$ with $t \neq t^{\prime}$, then this implies that $\frac{g\left(t^{\prime}\right)-g(t)}{f\left(t^{\prime}\right)-f(t)}=$ -1 , which contradicts to the condition $\left|\frac{g(t+\delta t)-g(t)}{f(t+\delta t)-f(t)}\right|<1$ for all $t$ and $\delta t$. Therefore, $\varphi$ is injective and likewise, we can show that $\psi$ is injective. Since $\varphi$ and $\psi$ are continuous bijections from $\mathbb{R}$ to $\mathbb{R}$, the topological domain of invariance implies that $\varphi$ and $\psi$ are homeomorphisms.

The equation $\varphi\left(t_{2}\right)-\varphi\left(t_{1}\right)=\left(f\left(t_{2}\right)-f\left(t_{1}\right)\right)\left[1+\frac{g\left(t_{2}\right)-g\left(t_{1}\right)}{f\left(t_{2}\right)-f\left(t_{1}\right)}\right]$ tells us that, by the condition $\left|\frac{g(t+\delta t)-g(t)}{f(t+\delta t)-f(t)}\right|<1, \varphi$ is increasing if and only if $f$ is increasing. Likewise, we can show that $\psi$ is increasing if and only if $f$ is increasing.

By simple calculation, we can show that $F$ has the desired form when expressed in terms of $\varphi$ and $\psi$. This completes the proof of the first part.

To prove the converse, let $\varphi$ and $\psi$ be increasing homeomorphisms on $\mathbb{R}$. Let $f=\frac{1}{2}(\varphi+\psi)$ and $g=\frac{1}{2}(\varphi-\psi)$. Then $g$ is continuous and $f$ is an increasing homeomorphism. Since $\varphi$ and $\psi$ are homeomorphisms, we have $\sup (f+g)=\sup \varphi=\infty, \sup (f-g)=\sup \psi=\infty, \inf (f+g)=\inf \varphi=-\infty$ and $\inf (f-g)=\inf \psi=-\infty$.

We now show that $f$ and $g$ satisfy the inequality $\left|\frac{g(t+\delta t)-g(t)}{f(t+\delta t)-f(t)}\right|<1$. Let $\Delta=\frac{g(t)-g\left(t_{0}\right)}{f(t)-f\left(t_{0}\right)}=\frac{\varphi(t)-\varphi\left(t_{0}\right)-\left(\psi(t)-\psi\left(t_{0}\right)\right)}{\varphi(t)-\varphi\left(t_{0}\right)+\psi(t)-\psi\left(t_{0}\right)}$. Without loss of generality, we can assume $t>t_{0}$ and it is easy to see that $-1<\Delta<1$, since $\varphi$ and $\psi$ are increasing. Therefore, by the theorem 2.1, the function $F$ defined as in the statement, is a causal automorphism. By the exactly same argument, we can show that the assertion also holds when $\varphi$ and $\psi$ are both decreasing homeomorphisms. 


\section{The group of CAUSAl AUtomorphisms on $\mathbb{R}_{1}^{2}$}

In this section, we denote the group of all causal automorphisms on $\mathbb{R}_{1}^{2}$ by $G$, and we analyze its group structure. For this we let $H(\mathbb{R})$ be the group of all homeomorphisms on $\mathbb{R}$ and let $H=H^{+} \cup H^{-}$where $H^{+}=\{(\varphi, \psi) \in H(\mathbb{R}) \times H(\mathbb{R}) \mid \varphi, \psi$ are increasing $\}$ and $H^{-}=\{(\varphi, \psi) \in$ $H(\mathbb{R}) \times H(\mathbb{R}) \mid \varphi, \psi$ are decreasing $\}$. Then $H$ is a subgroup of $H(\mathbb{R}) \times H(\mathbb{R})$ under the operation induced from $H(\mathbb{R}) \times H(\mathbb{R})$.

From the Theorem 2.2 , we can see that any causal automorphism $F$ on $\mathbb{R}_{1}^{2}$ corresponds to a unique element in $H$ and, conversely, each elements in $H$ uniquely determines a causal automorphism on $\mathbb{R}_{1}^{2}$. Thus, there exists a one-to-one correspondence between $G$ and $H$ as a set. It might seem that $G$ is isomorphic to $H$. However, we cannot obtain an isomorphism in this way and we define a new operation on $H$ as follows.

We define a $\mathbb{Z}_{2}$-action on $H$ by $a \cdot(\varphi, \psi)=(\varphi, \psi)$ if $a=0$ and $a$. $(\varphi, \psi)=(\psi, \varphi)$ if $a=1$. If we define a map $\pi: H \rightarrow \mathbb{Z}_{2}$ by $\pi(x)=0$ if $x \in H^{+}$and $\pi(x)=1$ if $x \in H^{-}$, then $\pi$ is a group homomorphism with $H$ equipped with the operation induced from $H(\mathbb{R}) \times H(\mathbb{R})$. Note that $\pi(\varphi, \psi)=\pi\left(\varphi^{-1}, \psi^{-1}\right)=\pi(\psi, \varphi)$ and $\pi(\pi(a, b) \cdot(\varphi, \psi))=\pi(\varphi, \psi)$ for any $(a, b) \in H$.

To get an isomorphism from $G$ to $H$, we define a new operation $*$ on $H$ by $(\alpha, \beta) *(\varphi, \psi)=(\alpha, \beta) \circ \pi(\alpha, \beta) \cdot(\varphi, \psi)$ where $\cdot$ is the $\mathbb{Z}_{2}$-action defined above and $\circ$ is the operation induced from $H(\mathbb{R}) \times H(\mathbb{R})$.

Theorem 3.1. The set $H$ under $*$ is a group and is isomorphic to $G$.

Proof. To show associativity, by calculation, we have, $(a, b) *\{(c, d) *(e, f)\}=$ $(a, b) *\{(c, d) \circ \pi(c, d) \cdot(e, f)\}=(a, b) \circ \pi(a, b) \cdot\{(c, d) \circ \pi(c, d) \cdot(e, f)\}$. Thus, we have the following four cases.

\begin{tabular}{|c|c|c|}
\hline$\pi(a, b)$ & $\pi(c, d)$ & $(a, b) *\{(c, d) *(e, f)\}$ \\
\hline 0 & 0 & $(a, b) \circ\{(c, d) \circ(e, f)\}=(a c e, b d f)$ \\
\hline 0 & 1 & $(a, b) \circ\{(c, d) \circ(f, e)\}=(a c f, b d e)$ \\
\hline 1 & 0 & $(a, b) \circ(d f, c e)=(a d f, b c e)$ \\
\hline 1 & 1 & $(a, b) \circ(d e, c f)=($ ade,$b c f)$ \\
\hline
\end{tabular}

On the other hand, we have

$$
\begin{aligned}
\{(a, b) *(c, d)\} *(e, f) & =\{(a, b) \circ \pi(a, b) \cdot(c, d)\} *(e, f) \\
& =\{(a, b) \circ \pi(a, b) \cdot(c, d)\} \circ \pi((a, b) \circ \pi(a, b) \cdot(c, d)) \cdot(e, f) \\
& =\{(a, b) \circ \pi(a, b) \cdot(c, d)\} \circ\{\pi(a, b) \pi(\pi(a, b) \cdot(c, d))\} \cdot(e, f)
\end{aligned}
$$


$(\because \pi$ is a group homomorphism.)

$$
=\{(a, b) \circ \pi(a, b) \cdot(c, d)\} \circ\{\pi(a, b) \pi(c, d)\} \cdot(e, f)
$$

$(\because \pi(\pi(a, b) \cdot(c, d))=\pi(c, d))$

Thus, we have the following four cases.

\begin{tabular}{|c|c|c|}
\hline$\pi(a, b)$ & $\pi(c, d)$ & $\{(a, b) *(c, d)\} *(e, f)$ \\
\hline 0 & 0 & $\{(a, b) \circ(c, d)\} \circ(e, f)=(a c e, b d f)$ \\
\hline 0 & 1 & $\{(a, b) \circ(c, d)\} \circ(f, e)=(a c f, b d e)$ \\
\hline 1 & 0 & $\{(a, b) \circ(d, c)\} \circ(f, e)=(a d f, b c e)$ \\
\hline 1 & 1 & $\{(a, b) \circ(d, c)\} \circ(e, f)=(a d e, b c f)$ \\
\hline
\end{tabular}

The above two tables tell us that the operation $*$ satisfies the associative law.

The following two formulae show that $(i d, i d)$ is the identity element in $H$ under $*$.

$$
\begin{aligned}
(i d, i d) *(\varphi, \psi) & =(i d, i d) \circ \pi(i d, i d) \cdot(\varphi, \psi) \\
& =(i d, i d) \circ(\varphi, \psi)=(\varphi, \psi), \\
(\varphi, \psi) *(i d, i d) & =(\varphi, \psi) \circ \pi(\varphi, \psi) \cdot(i d, i d) \\
& =(\varphi, \psi) \circ(i d, i d)=(\varphi, \psi) .
\end{aligned}
$$

The following two formulae show that, for given $(\varphi, \psi) \in H, \pi(\varphi, \psi)$. $\left(\varphi^{-1}, \psi^{-1}\right)$ is the inverse element of $(\varphi, \psi)$.

$$
\begin{aligned}
(\varphi, \psi) *\left\{\pi(\varphi, \psi) \cdot\left(\varphi^{-1}, \psi^{-1}\right)\right\} & =(\varphi, \psi) \circ \pi(\varphi, \psi) \cdot\left\{\pi(\varphi, \psi) \cdot\left(\varphi^{-1}, \psi^{-1}\right)\right\} \\
& =(\varphi, \psi) \circ\{\pi(\varphi, \psi) \pi(\varphi, \psi)\} \cdot\left(\varphi^{-1}, \psi^{-1}\right) \\
& =(\varphi, \psi) \circ\left(\varphi^{-1}, \psi^{-1}\right)=(i d, i d), \\
\left\{\pi(\varphi, \psi) \cdot\left(\varphi^{-1}, \psi^{-1}\right)\right\} *(\varphi, \psi) & =\left\{\pi(\varphi, \psi) \cdot\left(\varphi^{-1}, \psi^{-1}\right)\right\} \circ\left\{\pi\left(\pi(\varphi, \psi) \cdot\left(\varphi^{-1}, \psi^{-1}\right)\right)\right\} \cdot(\varphi, \psi) \\
& =\left\{\pi(\varphi, \psi) \cdot\left(\varphi^{-1}, \psi^{-1}\right)\right\} \circ\{\pi(\varphi, \psi) \cdot(\varphi, \psi)\}=(i d, i d) .
\end{aligned}
$$

Therefore, $H$ forms a group under the operation $*$.

We now show that the groups $G$ and $H$ under $*$ are isomorphic. For given causal automorphism $F(x, y)=\left(F_{1}(x, y), F_{2}(x, y)\right)$, Theorem2.2 tells us that $F_{1}+F_{2}$ and $F_{1}-F_{2}$ determine unique element in $H$. Therefore, we can define $\Pi: G \rightarrow H$ by

$\Pi(F)=$ (homeomorphism determined by $F_{1}+F_{2}$, homeomorphism determined by $\left.F_{1}-F_{2}\right)$ 
By Theorem 2.2. $\Pi$ is a bijection and we only need to show that $\Pi$ is a homomorphism. We have the following four cases.

i) Let $G=\frac{1}{2}(\alpha(x+y)+\beta(x-y), \alpha(x+y)-\beta(x-y))$ and $F=\frac{1}{2}(\varphi(x+y)+$ $\psi(x-y), \varphi(x+y)-\psi(x-y))$ where both $(\alpha, \beta)$ and $(\varphi, \psi)$ are in $H^{+}$. Then, we have $G \circ F(x, y)=\frac{1}{2}(\alpha \circ \varphi(x+y)+\beta \circ \psi(x-y), \alpha \circ \varphi(x+y)-\beta \circ \psi(x-y))$ and thus $\Pi(G \circ F)=(\alpha \circ \varphi, \beta \circ \psi)$. Since $\Pi(G)=(\alpha, \beta)$ and $\Pi(F)=(\varphi, \psi)$, we have $\Pi(G \circ F)=\Pi(G) * \Pi(F)$.

ii) Let $G=\frac{1}{2}(\alpha(x+y)+\beta(x-y), \alpha(x+y)-\beta(x-y))$ and $F=$ $\frac{1}{2}(\varphi(x-y)+\psi(x+y), \varphi(x-y)-\psi(x+y))$ where $(\alpha, \beta)$ is in $H^{+}$and $(\varphi, \psi)$ is in $H^{-}$. Then, we have $G \circ F(x, y)=\frac{1}{2}(\alpha \circ \varphi(x-y)+\beta \circ \psi(x+$ $y), \alpha \circ \varphi(x-y)-\beta \circ \psi(x+y))$ and thus $\Pi(G \circ F)=(\alpha \circ \varphi, \beta \circ \psi)$. Since $\Pi(G)=(\alpha, \beta)$ and $\Pi(F)=(\varphi, \psi)$, we have $\Pi(G \circ F)=\Pi(G) * \Pi(F)$.

iii) Let $G=\frac{1}{2}(\alpha(x-y)+\beta(x+y), \alpha(x-y)-\beta(x+y))$ and $F=$ $\frac{1}{2}(\varphi(x+y)+\psi(x-y), \varphi(x+y)-\psi(x-y))$ where $(\alpha, \beta)$ is in $H^{-}$and $(\varphi, \psi)$ is in $H^{+}$. Then, we have $G \circ F(x, y)=\frac{1}{2}(\alpha \circ \psi(x-y)+\beta \circ \varphi(x+$ $y), \alpha \circ \psi(x-y)-\beta \circ \varphi(x+y))$ and thus $\Pi(G \circ F)=(\alpha \circ \psi, \beta \circ \varphi)$. Since $\Pi(G)=(\alpha, \beta)$ and $\Pi(F)=(\varphi, \psi)$, we have $\Pi(G \circ F)=(\alpha \circ \psi, \beta \circ \varphi)=$ $(\alpha, \beta) \circ \pi(\alpha, \beta) \cdot(\varphi, \psi)=\Pi(G) * \Pi(F)$.

iv) Let $G=\frac{1}{2}(\alpha(x-y)+\beta(x+y), \alpha(x-y)-\beta(x+y))$ and $F=\frac{1}{2}(\varphi(x-y)+$ $\psi(x+y), \varphi(x-y)-\psi(x+y))$ where both $(\alpha, \beta)$ and $(\varphi, \psi)$ are in $H^{-}$. Then, we have $G \circ F(x, y)=\frac{1}{2}(\alpha \circ \psi(x+y)+\beta \circ \varphi(x-y), \alpha \circ \psi(x+y)-\beta \circ \varphi(x-y))$ and thus $\Pi(G \circ F)=(\alpha \circ \psi, \beta \circ \varphi)$. Since $\Pi(G)=(\alpha, \beta)$ and $\Pi(F)=(\varphi, \psi)$, we have $\Pi(G \circ F)=(\alpha \circ \psi, \beta \circ \varphi)=(\alpha, \beta) \circ \pi(\alpha, \beta) \cdot(\varphi, \psi)=\Pi(G) * \Pi(F)$.

This show that $\Pi$ is an isomorphism and the proof is completed.

In Ref. 3, it is shown that $H(\mathbb{R})$ is a subgroup of $G$ and this can also be seen in the above theorem as follows. If we define a map $\Omega: H(\mathbb{R}) \rightarrow H$ by $\Omega(f)=(f, f)$, then it is easy to see that $\Omega$ is an injective homomorphism and thus, $H(\mathbb{R})$ is a subgroup of $G$ through an injective homomorphism $\Pi^{-1} \circ \Omega$. Zeeman's result tells us that the group of causal automorphisms on $\mathbb{R}_{1}^{n}$ is finite dimensional when $n \geq 3$ and our result tells us that the group is infinite dimensional when $n=2$.

\section{ACKNOWLEDGEMENT}

This work was supported by a grant from the College of Applied Science, Kyung Hee University research professor fellowship. 


\section{REFERENCES}

[1] E.C. Zeeman, Causality implie the Lorentz group, J. Math. Phys. 5, (1964) pp.490.

[2] D.-H. Kim, Causal automorphisms of two-dimensional Minkowski space-time, Class. Quantum. Grav. To appear

[3] D.-H. Kim, An imbedding of Lorentzian manifolds, Class. Quantum. Grav. 26, (2009) pp. 075004.

Department of Applied Mathematics, College of Applied Science, Kyung Hee University, Seocheon-dong, Giheung-Gu,Yongin-si, Gyeonggi-do 446-701, REPUblic OF Korea

E-mail address: mathph@khu.ac.kr 